Pacific Journal of Mathematics

SOME NONOSCILLATION CRITERIA FOR HIGHER ORDER 


\title{
SOME NONOSCILLATION CRITERIA FOR HIGHER ORDER NONLINEAR DIFFERENTIAL EQUATIONS
}

\author{
JOHN R. GRAEF
}

\begin{abstract}
Sufficient conditions for an $n$th order nonlinear differential equation to be nonoscillatory are given. An essential part of the hypotheses is that a related linear equation be disconjugate.
\end{abstract}

The linear differential equation

$$
x^{(n)}+p(t) x=0
$$

where $p:\left[t_{0}, \infty\right) \rightarrow R$ is continuous, is said to be eventually disconjugate if there exists $T \geqq t_{0}$ such that no solution of (1) has more than $n-1$ zeros (counting multiplicities) on [T, ). A solution $x(t)$ of (1) (or equation (2) below) will be called nonoscillatory if there exists $t_{1} \geqq t_{0}$ such that $x(t) \neq 0$ for $t \geqq t_{1}$. Equation (1) (or (2)) will be called nonoscillatory if all its solutions are nonoscillatory. Clearly, disconjugacy implies nonoscillation. On the other hand, for $n=2,3$ or 4 and either $p(t)>0$ or $p(t)<0$, if equation (1) is nonoscillatory, then (1) is eventually disconjugate. Whether this is true for $n>4$ remains an open question (see Nehari [11]).

In this paper we consider the nonlinear differential equation

$$
x^{(n)}+q(t) f\left(t, x, x^{\prime}, \cdots, x^{(n-1)}\right)=0
$$

where $q:\left[t_{0}, \infty\right) \rightarrow R$ and $f:\left[t_{0}, \infty\right) \times R^{n} \rightarrow R$ are continuous, and obtain some nonoscillation results by making assumptions on the disconjugacy of certain related linear equations. A discussion of disconjugacy criteria for linear differential equations can be found in Coppel [2], Levin [10], Nehari [11], Trench [12], or Willett [13]. For a discussion of nonoscillation criteria for second order nonlinear equations we refer the reader to the recent papers of Coffman and Wong [1], Graef and Spikes [3-5], Wong [14], and the references contained therein. There appears to be no known sufficient conditions for nonoscillation of higher order nonlinear equations.

We will assume that there is a continuous function $W:\left[t_{0}, \infty\right) \times$ $R^{n} \rightarrow R$ such that

$$
\left|f\left(t, u_{1}, \cdots, u_{n}\right)\right| \leqq W\left(t, u_{1}, \cdots, u_{n}\right)\left|u_{1}\right|
$$

for all $\left(t, u_{1}, \cdots, u_{n}\right) \in\left[t_{0}, \infty\right) \times R^{n}$, and 


$$
f\left(t, u_{1}, \cdots, u_{n}\right) / u_{1} \rightarrow A \text { as } u_{1} \rightarrow 0
$$

THEOREM 1. Suppose that conditions (3) and (4) hold, $W\left(t, u_{1}, \cdots, u_{n}\right) \leqq B$ and $M=\max \{|A|, B\}$. If the equations

$$
x^{(n)} \pm M|q(t)| x=0
$$

are eventually disconjugate, then equation (2) is nonoscillatory.

Proof. Suppose that equations (5) are disconjugate on $[T, \infty)$ where $T \geqq t_{0}$ and let $x(t)$ be a solution of (1). Define $Q:[T, \infty) \rightarrow R$ by

$$
Q(t)= \begin{cases}q(t) f\left(t, x(t), \cdots, x^{(n-1)}(t)\right) / x(t), & \text { if } x(t) \neq 0 \\ A q(t), & \text { if } x(t)=0 .\end{cases}
$$

It then follows that $Q(t)$ is continuous and $x(t)$ is a solution of

$$
x^{(n)}+Q(t) x=0
$$

Kondrat'ev [9] showed that if $p_{1}(t) \leqq p_{2}(t)$ and the equations

$$
x^{(n)}+p_{1}(t) x=0, \quad i=1,2
$$

are disconjugate on $[T, \infty)$, then for any $p(t)$ with $p_{1}(t) \leqq p(t) \leqq p_{2}(t)$ the equation

$$
x^{(n)}+p(t) x=0
$$

is disconjugate on $[T, \infty)$. Here we have $|Q(t)| \leqq M|q(t)|$ so - $M|q(t)| \leqq Q(t) \leqq M|q(t)|$. Hence equation (6) is disconjugate and so $x(t)$ is nonoscillatory.

REMARK 1 . If $q(t) \geqq 0$ and $u_{1} f\left(t, u_{1}, \cdots, u_{n}\right) \geqq 0$, then $Q(t) \geqq$ 0 . Since the equation $x^{(n)}=0$ is disconjugate on $[T, \infty)$ for any $T \geqq t_{0}$, we would only need to assume that equation (5) with " + " is eventually disconjugate. Note also that condition (4) is only needed to insure that $Q$ is continuous.

REMARK 2. Equations (5) are eventually disconjugate if, for example,

$$
\int_{t_{0}}^{\infty} t^{n-1}|q(t)| d t<\infty
$$


(see Kiguradze [8], Kondrat'ev [9], or Willett [13]). In this regard we would then have a generalization of a result of Kartsatos [7; Theorem 2].

Willett [13; Theorem 1.4] has shown that if for each $i=1,2, \cdots, n$, $p_{i}:\left[t_{0}, \infty\right) \rightarrow R$ is continuous and

$$
\int_{t_{0}}^{\infty} t^{t-1}\left|p_{\imath}(t)\right| d t<\infty
$$

then the equation

$$
x^{(n)}+p_{1}(t) x^{(n-1)}+\cdots+p_{n}(t) x=0
$$

is eventually disconjugate. (Recently Gustafson [6] showed that even though nonoscillation implies disconjugacy for equation (8) with $n=2$, this is not the case for $n>2$.) Employing the method of proof used above we can obtain that all solutions of

$$
x^{(n)}+p_{1}(t) f_{1}\left(x^{(n-1)}\right)+\cdots+p_{n}(t) f_{n}(x)=0
$$

are nonoscillatory.

THEOREM 2. Suppose that condition (7) holds and there are bounded continuous functions $W_{i}:\left[t_{0}, \infty\right) \rightarrow R, i=1,2, \cdots, n$ such that

$$
\left|f_{t}(u)\right| \leqq W_{\imath}(u)|u|
$$

and

$$
f_{\imath}(u) / u \rightarrow A_{\imath} \quad \text { as } \quad u \rightarrow 0 .
$$

Then all solutions of (9) are nonoscillatory.

Proof. If $x(t)$ is a solution of (9), then $x(t)$ is also a solution of

$$
x^{(n)}+Q_{1}(t) x^{(n-1)}+\cdots+Q_{n}(t) x=0
$$

where

$$
Q_{i}(t)= \begin{cases}p_{i}(t) f_{l}\left(x^{(n-i)}(t)\right) / x^{(n-i)}(t), & \text { if } x^{(n-i)}(t) \neq 0 \\ A_{i} p_{\imath}(t), & \text { if } x^{(n-i)}(t)=0\end{cases}
$$

In addition, for each $i=1,2, \cdots, n$ 


$$
\begin{aligned}
\int_{t_{0}}^{\infty} t^{t-1}\left|Q_{i}(t)\right| d t & \leqq \int_{t_{0}}^{\infty} t^{i-1}\left[\left|p_{t}(t)\right|\left|f_{\imath}\left(x^{(n-i)}(t)\right)\right| /\left|x^{(n-t)}(t)\right|\right] d t \\
& \leqq \int_{t_{0}}^{\infty} t^{i-1}\left|p_{t}(t)\right| W_{i}\left(x^{(n-t)}(t)\right) d t \\
& \leqq K_{i} \int_{t_{0}}^{\infty} t^{t-1}\left|p_{t}(t)\right| d t \\
& <\infty
\end{aligned}
$$

where $K_{r}$ is a constant. It follows from Willett's theorem that equation (10) is disconjugate and hence $x(t)$ is nonoscillatory.

Clearly various other forms of equation (9) can be handled in a similar fashion.

As an example of the above results, consider the equation

$$
x^{(n)}+x^{3}(\sin t) / t^{n+1}\left(x^{2}+1\right)=0 .
$$

The corresponding linear equation

$$
x^{(n)}+x(\sin t) / t^{n+1}=0
$$

is disconjugate, so equation (11) is nonoscillatory.

\section{REFERENCES}

1. C. V. Coffman and J. S. W. Wong, Oscillation and nonoscillation theorems for second order ordinary differential equations, Funkcial. Ekvac., 15 (1972), 119-130.

2. W. A. Coppel, Disconjugacy, Lecture Notes in Math., 220, Springer-Verlag, New York, 1971.

3. J. R. Graef and P. W. Spikes, A nonoscillation result for second order ordinary differential equations, Rend. Accad. Sci. Fis. Mat. Napoli, (4) 41 (1974), 3-12.

4. - Nonoscllation theorems for forced second order nonlinear differential equations, Atti Accad. Naz. Lincei Kend. Cl. Sci. Fis. Mat. Natur., to appear.

5. - Sufficient conditions for nonoscillation of a second order nonlinear differential equation, Proc. Amer. Math. Soc., 50 (1975), 289-292.

6. G. B. Gustafson, The nonequivalence of oscillation and nondisconjugacy, Proc. Amer. Math. Soc., 25 (1970), 254-260.

7. A. G. Kartsatos, Maintenance of oscillations under the effect of a periodic forcing term, Proc. Amer. Math. Soc., 33 (1972), 377-383.

8. I. T. Kiguradze, Oscillation properties of solutions of certain ordinary differential equations, Soviet Math. Dokl., 3 (1962), 649-652.

9. V. A. Kondrat'ev, Oscillatory properties of solutions of the equation $y^{(n)}+p(x) y=0$, Trudy Moskov. Mat. Obšč., 10 (1961), 419-436.

10. A. Ju. Levin, Non-oscillation of solutions of the equation $x^{(n)}+p_{1}(t) x^{(n-1)}+\cdots+p_{n}(t) x=0$, Russian Math. Surveys, 24 (1969), 43-99.

11. Z. Nehari, Nonlinear techniques for linear oscillation problems, Trans. Amer. Math. Soc., 210 (1975), 387-406. 
12. W. F. Trench, A sufficient condition for eventual disconjugacy, Proc. Amer. Math. Soc., 52 (1975), 139-146.

13. D. Willett, Disconjugacy tests for singular linear differential equations, SIAM J. Math. Anal., 2 (1971), 536-545.

14. J. S. W. Wong, On the generalized Emden-Fowler equation, SIAM Review, 17 (1975), 339-360.

Received March 15, 1976. Research supported by the Mississippi State University Biological and Physical Sciences Research Institute.

MisSisSIPPI STATE UNIVERSITY 




\section{Pacific Journal of Mathematics}

\section{Vol. 66, No. $1 \quad$ November, 1976}

Helen Elizabeth. Adams, Factorization-prime ideals in integral domains ............ Patrick Robert Ahern and Robert Bruce Schneider, The boundary behavior of Henkin's kernel.

Daniel D. Anderson, Jacob R. Matijevic and Warren Douglas Nichols, The Krull

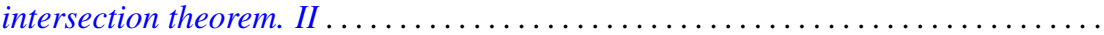

Efraim Pacillas Armendariz, On semiprime P.I.-algebras over commutative regular

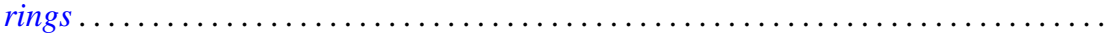

Robert H. Bird and Charles John Parry, Integral bases for bicyclic biquadratic fields

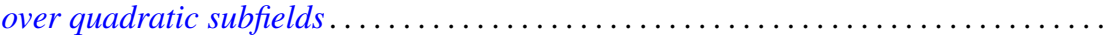

Tae Ho Choe and Young Hee Hong, Extensions of completely regular ordered

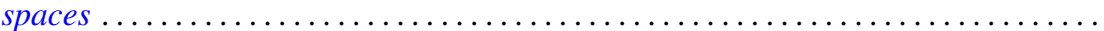

John Dauns, Generalized monoform and quasi injective modules ...............

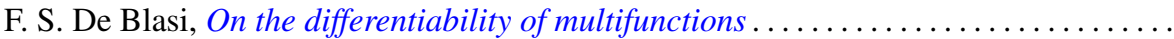

Paul M. Eakin, Jr. and Avinash Madhav Sathaye, R-endomorphisms of $R[[X]]$ are

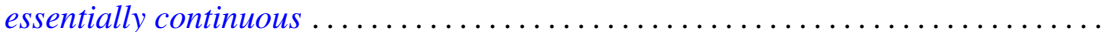

Larry Quin Eifler, Open mapping theorems for probability measures on metric

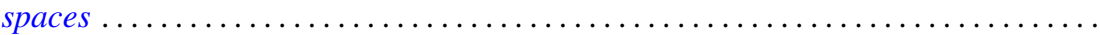

Garret J. Etgen and James Pawlowski, Oscillation criteria for second order self adjoint

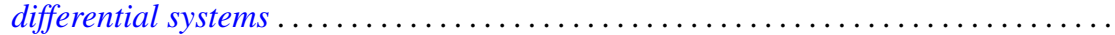

Ronald Fintushel, Local $S^{1}$ actions on 3-manifolds .

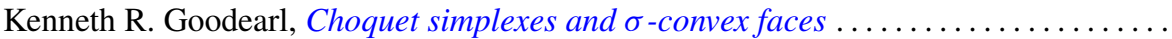

John R. Graef, Some nonoscillation criteria for higher order nonlinear differential

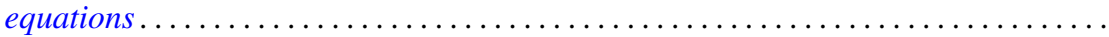

Charles Henry Heiberg, Norms of powers of absolutely convergent Fourier series: an example.

Les Andrew Karlovitz, Existence of fixed points of nonexpansive mappings in a space

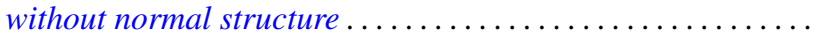

Gangaram S. Ladde, Systems of functional differential inequalities and functional

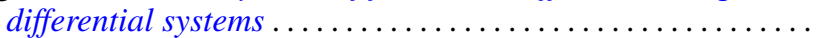

Joseph Michael Lambert, Conditions for simultaneous approximation and interpolation with norm preservation in $C[a, b]$.

Ernest Paul Lane, Insertion of a continuous function.

Robert F. Lax, Weierstrass points of products of Riemann surfaces .

Dan McCord, An estimate of the Nielsen number and an example concerning the Lefschetz fixed point theorem...

Paul Milnes and John Sydney Pym, Counterexample in the theory of continuous functions on topological groups...

Peter Johanna I. M. De Paepe, Homomorphism spaces of algebras of holomorphic functions

Judith Ann Palagallo, A representation of additive functionals on $L^{p}$-spaces,

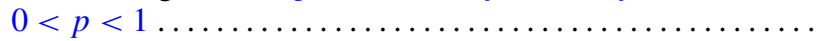

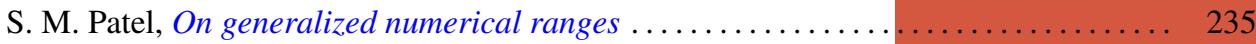

Thomas Thornton Read, A limit-point criterion for expressions with oscillatory

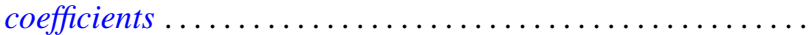

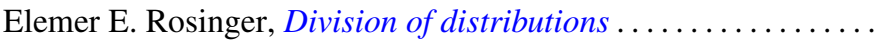

Peter S. Shoenfeld, Highly proximal and generalized almost finite

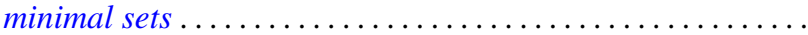

R. Sirois-Dumais and Stephen Willard, Quotient-universal sequential spaces

Robert Charles Thompson, Convex and concave functions of singular values of matrix sums....

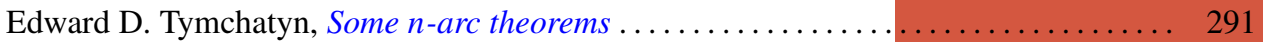

